\title{
Protective Effects of Bogijetong Decoction and Its Selected Formula on Neuropathic Insults in Streptozotocin-Induced Diabetic Animals
}

\author{
Ki-Joong Kim, Uk Namgung, and Chung Sik Cho \\ Department of Oriental Medicine, Daejeon University, Daejeon 300-716, Republic of Korea \\ Correspondence should be addressed to Chung Sik Cho; choolo2@dju.kr
}

Received 27 April 2017; Accepted 27 June 2017; Published 16 August 2017

Academic Editor: Shuang-En Chuang

Copyright (C) $2017 \mathrm{Ki}$-Joong Kim et al. This is an open access article distributed under the Creative Commons Attribution License, which permits unrestricted use, distribution, and reproduction in any medium, provided the original work is properly cited.

Bogijetong decoction (BGJTD) is a mixture of herbal formulation which is used in the traditional Korean medicine for the treatment of neuropathic pain caused by diabetes. Here, we investigated the regulatory effects of BGJTD and its reconstituted decoction subgroups on the neuropathic responses in streptozotocin- (STZ-) induced diabetic animals. Be decoction (BeD) was formulated by selecting individual herbal components that induced neurite outgrowth most efficiently in each subgroup. BeD induced the neurite outgrowth in DRG neurons most efficiently among decoction subgroups and downregulated the production of TNF- $\alpha$ from the sciatic nerves in STZ-diabetic animals. While the levels of phospho-Erk1/2 were elevated in the sciatic nerves of STZdiabetic animals by BGJTD and BeD treatments, p38 level was downregulated by BGJTD and BeD. A single herbal component of $\mathrm{BeD}$ induced neurite outgrowth comparable to $\mathrm{BeD}$ and was involved in the regulation of Erk1/2 activation and TNF- $\alpha$ production in DRG neurons. Oral administration of BGJTD and BeD in STZ-diabetic animals reduced the latency time responding to thermal stimulation. Our results suggest that the reconstituted formulation is as effective as conventional BGJTD in inducing biochemical and behavioral recoveries from the neuropathy in peripheral nerves and thus the experimental reductionism may be applied to develop the methodology for compositional analysis of herbal decoctions.

\section{Background}

Diabetes is a disease causing high mortality and morbidity with complications and represents $5 \%$ of prevalence worldwide and $12-14 \%$ of prevalence in the United States in 2011-2012 [1]. Diabetes shows clinical presentation in major body organs such as kidneys, heart, pancreas, and eyes and thus results in diverse pathological symptoms and functional disturbances.

Of several animal models that have been developed to study type 1 and type 2 diabetes, streptozotocin- (STZ-) induced diabetic model is widely used because the experimental procedure is simple to induce hyperglycemia in studying type 1 diabetes and the procedure can also be used as an animal model for type 2 diabetes by feeding with high fat diet [2]. STZ, a glucosamine-nitrosourea compound, is transported preferentially into the pancreatic cells through the glucose transporter 2 (GLU2), in which it results in DNA damage that leads to the inhibition of insulin production [3]. Hyperglycemia is easily induced within several days after STZ injection and maintained for several months, which is thus useful to investigate diabetes-related complications such as peripheral neuropathy. Hyperglycemia causes metabolic disturbances such as unregulated increases in sorbitol and fructose as well as glucose in the cytoplasm of affected cells and increases osmotic stress to cells, causing the induction of signaling pathways such as MAP kinase pathway [4]. Antidepressants such as tricyclic compounds and SSRIs and anticonvulsants are used to alleviate diabetic neuropathic pain $[5,6]$. In the peripheral nerves, physiological functions of Schwann cells and axons might be affected in STZdiabetic animals, but the underlying mechanisms on cellular responses remain to be investigated.

BGJTD is the herbal formulation which was developed to treat peripheral neuropathy as sequelae of diabetes [7]. Previously, we reported that BGJTD administration into 
rodents was effective in attenuating the peripheral nerve damage caused by taxol injection and crush injury to the sciatic nerve [8]. In STZ-diabetic animal model, oral administration of BGJTD improved the regenerative responses of sciatic nerve axons in terms of upregulation of the marker proteins of axonal regeneration such as axonal growthassociated protein 43 (GAP-43), cell division cycle 2 (Cdc2), and phospho-vimentin as well as distal elongation of regenerating axons [9]. Moreover, some of herbal components of BGJTD revealed protective effects on tissues and organs in STZ-diabetic animals. Notably, antioxidant activity and antiacetylcholinesterase activity of Chaenomeles sinensis [10], attenuating effects of the extract of Pueraria thunbergiana on diabetic nephropathy [11], and protective effects of Panax ginseng on hippocampal neurons, kidneys from nephropathy, and pancreatic cells [12-14] were demonstrated in STZdiabetic animal models.

While the previous studies indicate the potential efficacy of BGJTD to protect peripheral nerves from neuropathic insults, its compositional diversity imposes an obstacle to analytical studies because BGJTD is composed of as many as 18 different herbal components. Thus, we have begun our study by dividing BGJTD into 4 different decoction subgroups based on the traditional medicinal theory, constituted a new formulation composed of active herbal components selected from 4 subgroups, and investigated the effects on peripheral nerve responses in STZ-diabetic rodents. Our data show that a new formula is as effective as BGJTD in mediating the recovery of the peripheral nerves from neuropathic insults.

\section{Materials and Methods}

2.1. Herbal Drug Preparation. Key features of herbal drugs used in the present study have been described in our recent paper in which the information of purchase and quality control of BGJTD and the validation of chemical ingredients were specified in detail [8]. In the present study, 18 different herbal components of BYHWD were divided into 4 subgroups $\mathrm{Ba}, \mathrm{Bb}, \mathrm{Bc}$, and $\mathrm{Bd}$ based on the theoretical description of individual ingredients. The major consideration for subgrouping was flow of qi energy, holistic balance of yin and yang spirit in the body, and blood flow and pain control, as described in our previous study [8]. Herbal compositions of each subgroup decoction are listed below (scientific names and, in parenthesis, their abbreviations and herbal names). Ba decoction was composed with Astragalus membranaceus (AM; Astragali Radix), Panax ginseng C. A. Meyer (PG; Ginseng Radix), Epimedium koreanum Nakai (EK; Epimedii Herba), and Ciborium barometz J. Smith (CB; Cibotii Rhizoma) (weight ratio of $30: 4: 10: 10$ ); Bb with Angelica gigas (AG; Angelicae Gigantis Radix), Rehmannia glutinosa (RG; Rehmanniae Radix Preparat), Cnidium officinale Makino (CO; Cnidii Rhizoma), and Spatholobus suberectus Dunn (SS; Spatholobi Caulis) (weight ratio of $7.5: 10: 7.5: 12$ ); Bc with Prunus persica (PP; Persicae Semen), Paeonia lactiflora Pall (PL; Paeoniae Radix Rubra), Carthamus tinctorius (CT; Carthami Flos), Lumbricidae (LB; Lumbricus), and Salvia miltiorrhiza (SM; Salviae Miltiorrhizae Radix) (weight ratio of $7.5: 7.5: 7.5: 5: 12)$, and Bd with Uncaria rhynchophylla (UR; Uncariae Ramulus et Uncus), Pueraria lobata Ohwi (PL; Puerariae Radix), Crassostrea gigas (CG; Crassostrea gigas), Albizia julibrissin Durazz (AJ; Albiziae Cortex), and Chaenomeles sinensis Koehne (CS; Chaenomelis Fructus) (weight ratio of $12: 8: 12: 12: 8$ ). Preparation of BGJTD and subgroup decoctions was essentially the same as described in our previous report [8], and final products in powders were stored at $-20^{\circ} \mathrm{C}$ or diluted in saline solution. Animals were treated with herbal drugs by oral administration $(400 \mathrm{mg} / \mathrm{kg}$, 0.1 and $0.5 \mathrm{ml}$ injection for mice and rats, resp.) on a daily basis for 2 weeks. Optimal dose was determined by analyzing dose response to injected BGJTD in STZ-diabetic mice (see below).

2.2. Experimental Animals and Preparation of Diabetic Animals. We used Sprague-Dawley rats (male, 200-250 g) and $\mathrm{Balb} / \mathrm{c}$ mice (male, 20-25g), which were purchased from Samtago (Seoul, Korea). They were maintained in animal rooms which were regulated at constant temperature $\left(22^{\circ} \mathrm{C}\right)$ and humidity (60\%) with a day and night cycle (lights on 7 am to $7 \mathrm{pm}$ ). We strictly observed the animal handling protocols regarding "the Animal-use Statement and Ethics Committee Approval Statement for Animal Experiments provided by Daejeon University (Daejeon, Korea)" as in our previous study [15]. STZ (Sigma, St. Louis, USA) was ip injected $(60 \mathrm{mg} / \mathrm{kg}$ for mouse and $45-50 \mathrm{mg} / \mathrm{kg}$ for rat) and blood glucose levels were measured by using a glucose meter (AccuChek, Roche Diagnostic, GmbH, Germany) before and after STZ injection and every 7 days up to 7 weeks. Animals showing higher than $300 \mathrm{mg} / \mathrm{dl}$ ( or $3 \mathrm{mg} / \mathrm{ml}$ ) of blood glucose at 1 week or 4 weeks after STZ injection were selected as diabetes-induced animals and used for the experiments. To determine optimal dose of herbal drug administration, mice were injected with STZ $(60 \mathrm{mg} / \mathrm{kg})$, and, 3 days later, animals showing $3 \mathrm{mg} / \mathrm{ml}$ of blood glucose or higher were selected and treated with different doses of BGJTD. Blood glucose levels were measured 7 days later. As shown in Figure 1(a), $400 \mathrm{mg} / \mathrm{kg}$ of BGJTD was the most effective in regulating glucose levels. We applied a dose of $400 \mathrm{mg} / \mathrm{kg}$ to other herbal drugs as well as BGJTD and used it for the rest of the present study.

2.3. Primary DRG Neuron Culture. Primary neurons were isolated from dorsal root ganglion (DRG) at lumbar levels 4 and 5 in adult rats. We prepared primary DRG neurons as described in our previous report [16]. Briefly, cells were dissociated and plated on the coverslips (Bellco, Glass Inc., Vineland, USA) which had been treated with solution of $0.01 \%$ poly-L-ornithine (Sigma) and laminin $(0.02 \mathrm{mg} /$ $\mathrm{ml}$, Collaborative Research, Bedford, USA) for overnight. Cells were cultured in Dulbecco's Modified Eagle's medium (DMEM) containing 5\% fetal bovine serum (GIBCO, Australia), $5 \%$ horse serum, $2 \mathrm{mM}$ glutamine, and $1 \%$ penicillinstreptomycin and incubated for 48 hours before the harvest for immunofluorescence staining. Primary antibodies used for immunofluorescence staining were anti-neurofilament 200 (NF-200, mouse monoclonal, rabbit polyclonal, 1:400, Sigma), anti-TNF- $\alpha$ (1:400, Rabbit polyclonal, Sigma), 


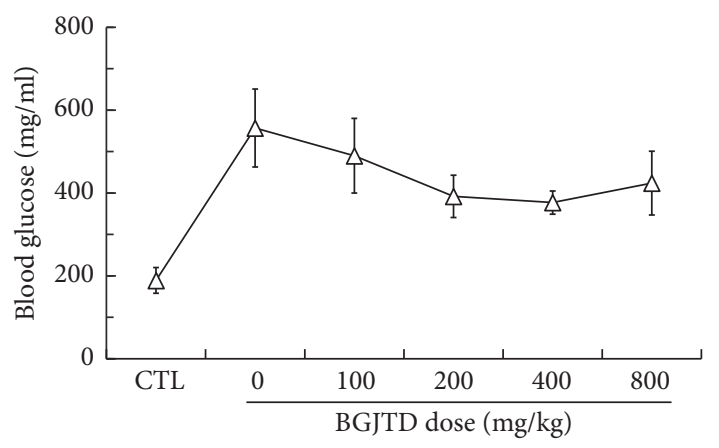

(a)

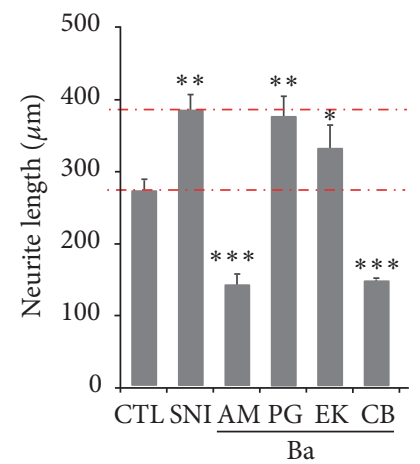

NF-200
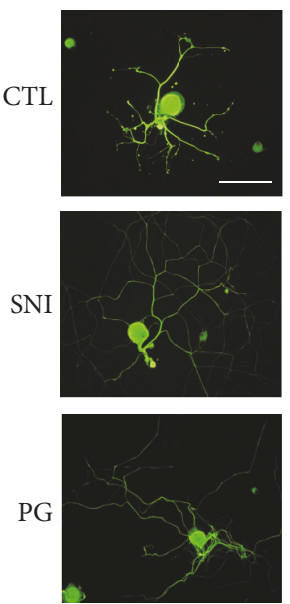

GAP-43
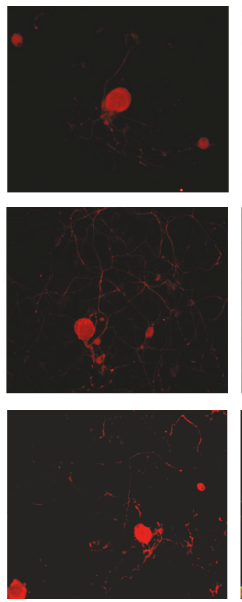
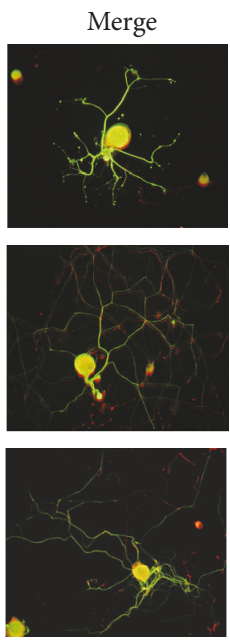

(c)

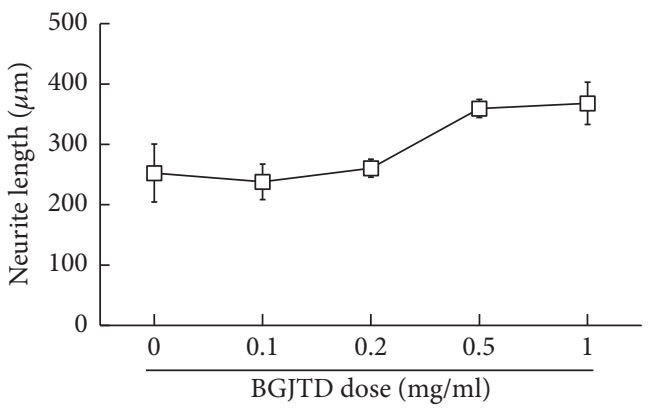

(b)

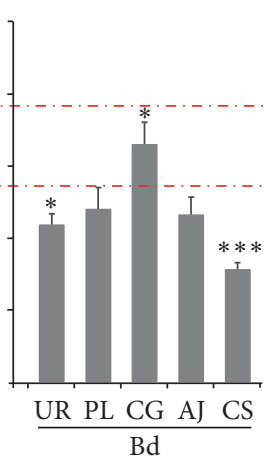

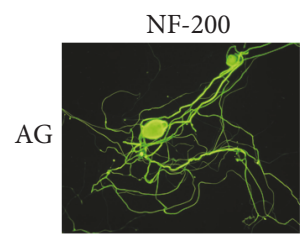
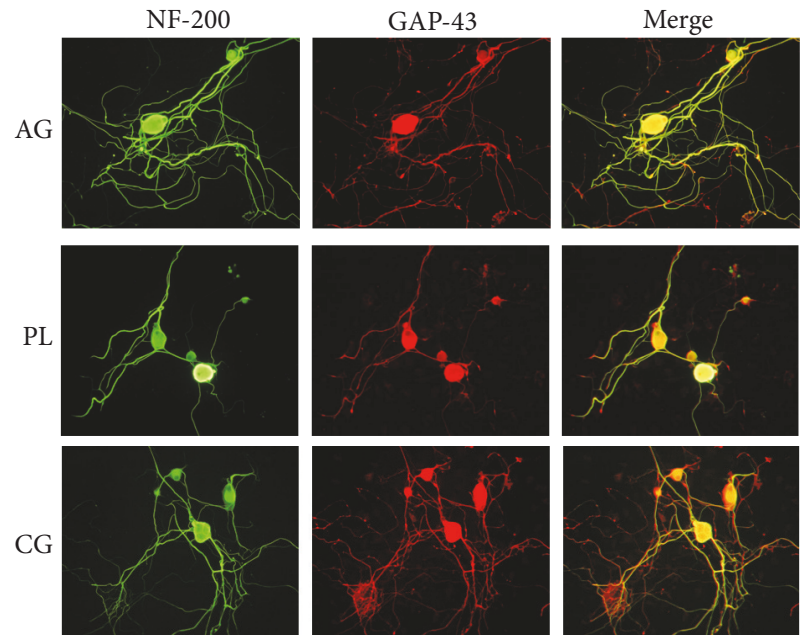

(d)

FIGURE 1: Dose responses to BGJTD and comparison of neurite outgrowth in DRG neurons after the treatment of individual constituents of BGJTD. (a) Changes of blood glucose levels with increasing doses of BGJTD. DGJTD up to $800 \mathrm{mg} / \mathrm{kg}$ was administered for 7 days to STZdiabetic mice. Number of animals per group $=4$. (b) Measurement of neurite length of DRG neurons in culture after the treatment of BGJTD. DRG neurons prepared from rats were cultured for $48 \mathrm{~h}$ in the presence of BGJTD at $0-1.0 \mathrm{mg} / \mathrm{ml}$, and neurite length was measured. Number of independent experiments $=4$. (c) Comparison of neurite outgrowth in individual subgroups Ba-Bd. Upper and lower red dotted lines denote the values of neurite length from animals given preconditioning nerve injury (SNI) and untreated control (CTL), respectively. Mean values of neurite length were compared among experimental groups (number of independent experiments $=4$ ). ${ }^{*} p<0.05,{ }^{* *} p<0.01,{ }^{* * *} p<$ 0.001 versus untreated control (CTL). Unabbreviated names of all herbal drugs are described in Materials and Methods. (d) Representative fluorescence images of DRG neurons treated with herbal drugs scoring the highest neurite extension from each subgroup, along with CTL and SNI groups. In (c) and (d), herbal drugs $(0.5 \mathrm{mg} / \mathrm{ml})$ were treated to DRG neurons for $48 \mathrm{~h}$ and harvested for immunofluorescence staining for NF-200 (green) and GAP-43 (red) signals. Scale bar in (d) $=100 \mu \mathrm{m}$. 
anti-phospho-Erk1/2 (1:400, rabbit polyclonal, Cell Signaling Tech, Boston, USA), and anti-GAP-43 (1:400, rabbit polyclonal, Santa Cruz Biotech. Dallas, USA) antibodies, and secondary antibodies were fluorescein-goat anti-mouse (1:400, Molecular Probes, Eugene, USA) and rhodaminegoat anti-rabbit antibodies (1:400, Invitrogen, Carlsbad, USA). Immunofluorescence images of cultured cells were analyzed by using fluorescence microscope and the digital images were captured and transferred to the Adobe Photoshop program (version 7.0). Length of neurites was measured by using i-Solution software program (Image and Microscope Technology, Goleta, USA). At least 20 neurons were randomly selected from the microscopic field and average length of neurites in each cell was determined. To determine optimal dose of herbal drugs in culture, DRG neurons were treated with different doses of BGJTD $(0.1-1.0 \mathrm{mg} / \mathrm{ml})$ and incubated for 48 hours before the harvest for immunofluorescence staining with anti-NF-200 antibody. Measurement of neurite length revealed that a concentration of $0.5 \mathrm{mg} / \mathrm{ml}$ of BGJTD was the most optimal (Figure 1(b)), and thus this dose was used for in vitro analyses of herbal drugs for the rest of present investigation.

2.4. Western Blot Analysis. Proteins expressed in the sciatic nerve were determined by western blot analysis. Sciatic nerves were rapidly dissected from rats and used for protein analysis. All the procedures including protein extraction, SDS-polyacrylamide gel electrophoresis (SDS-PAGE), and western blotting were essentially the same as described in our previous report [8]. Briefly, cells in the sciatic nerves were lysed using triton lysis buffer, sonicated, and centrifuged to collect the supernatant containing proteins. Protein $(15 \mu \mathrm{g})$ was used for SDS-PAGE (10\%) and western blotting analyses. Anti-phospho-Erk1/2 (1:1250, rabbit polyclonal, Cell Signaling, Seattle, USA), anti-p38 (1:1250, rabbit polyclonal, Santa Cruz Biotech.), anti-TNF- $\alpha$ (1:1250, rabbit polyclonal, Sigma), and anti-actin (1:10,000, mouse monoclonal, MP Biomedicals, Santa Ana, USA) antibodies were used as primary antibodies, and horseradish peroxidase- (HRP-) conjugated antibody $(1: 1250$; goat anti-rabbit or goat antimouse, Santa Cruz Biotech.) as a secondary antibody. Protein band intensity in the scanned images of X-ray film was determined by using the i-Solution software (Image \& Microscope Technology, Daejeon, Korea).

2.5. Immunofluorescence Staining of Nerve Sections. After in vivo experiments, sciatic nerves were rapidly isolated from rats and the longitudinal or transverse nerve sections $(20 \mu \mathrm{m})$ were prepared by using a cryostat (Leica CM1850, Germany). All the immunohistological procedures including fixation and permeabilization of sections, blocking, and primary and secondary antibody reactions were performed as previously described [8]. Primary antibodies used for immunofluorescence staining of nerve sections were anti-NF-200 (mouse monoclonal, 1:400, Sigma, St. Louis), anti-phosphoErk1/2 (1:400, rabbit polyclonal, Cell Signaling), anti-p38 (1:400, rabbit polyclonal, Santa Cruz Biotech.), and antiTNF- $\alpha$ (1:400, rabbit polyclonal) antibodies, and secondary antibodies were fluorescein-goat anti-mouse (1:400, rabbit polyclonal, Molecular Probes, Eugene, USA) and rhodaminegoat anti-rabbit (1:400, Invitrogen, Carlsbad, USA) antibodies. The nuclei in the nerve sections were visualized by staining with Hoechst dye $33258(2.5 \mu \mathrm{g} / \mathrm{ml})$ (bisbenzimide; Sigma). Immunofluorescence images in the sections were analyzed by using a Nikon fluorescence microscope, and digital images were transferred to the Adobe Photoshop program. For some analytical purpose, immunofluorescence images of two different colors were merged by using layerblending options in the Adobe Photoshop program.

\subsection{Real-Time PCR Polymerase Chain Reaction (RT-PCR).} We extracted total RNA from DRG at lumbar levels 4 and 5 in mice and performed real-time PCR to compare the expression levels of TNF- $\alpha$ mRNA among animal groups. In our recent report, we analyzed TNF- $\alpha$ mRNA in the spleen of LPS-injected mice and found the effects of acupuncture and vagotomy on TNF- $\alpha$ mRNA expression [15]. Here, realtime PCR for DRG RNA was performed essentially the same as described in the previous investigation. Quantification of TNF- $\alpha$ mRNA level relative to GAPDH control mRNA was performed by using a software converting program (Applied Biosystems) and the data were presented as fold changes to control mRNA expression.

2.7. Hot Plate Test. Herbal decoctions $(400 \mathrm{mg} / \mathrm{kg}$ ) or saline was orally administered to STZ-induced diabetic mice and was supplemented on a daily basis for 14 days. Thermal sensitivity of animals was measured by hot plate test as described previously [8] with a slight modification. Briefly, animals were initially adapted to a change in temperature by placing on a warm surface $\left(30^{\circ} \mathrm{C}\right)$ for $10 \mathrm{~min}$ and quickly transferred to a plate which was set to $50^{\circ} \mathrm{C}$ for $30 \mathrm{~s}$. Animal's behavior on a hot plate was recorded by a video camera, and data were transferred and saved into a computer. The latency to the lifting response of the right hind paw and the frequency to withdraw two hind legs from the plate were assessed. Behavioral scores in each animal were averaged by conducting the tests three times with a 10 min interval.

2.8. Statistical Analysis. Results are shown as mean \pm standard error of mean (SEM). Statistical analysis was evaluated by one-way ANOVA followed by Tukey's test post hoc analysis (SPSS computer software version 21.0), and statistical significance was reported as $p<0.05, p<0.01$, and $p<$ 0.001 .

\section{Results}

For 18 different herbal components of BGJTD, we divided them into four subgroup decoctions $\mathrm{Ba}, \mathrm{Bb}, \mathrm{Bc}$, and $\mathrm{Bd}$, based on the traditional medicinal theory. We treated cultured DRG neurons with individual herbal drugs and selected 4 drugs, one from each subgroup, that induced neurite outgrowth most efficiently. Neurons treated with these selected drugs, Panax ginseng (PG), Angelica gigas (AG), Paeonia lactiflora (PL), and Crassostrea gigas (CG), showed neurite outgrowth 
similar to that in the DRG neurons prepared from preconditioning sciatic nerve injury (SNI) (Figure 1(c)). Furthermore, cells treated with selected drugs revealed intense GAP-43 signals in the neuritic processes as well as in the cell body (Figure 1(d)). Thus, the fifth subgroup Be decoction (BeD) composed of PG, AG, PL, and CG was used for the analysis of neural responses together with the other subgroups. Glucose levels in STZ-injected animals were maintained higher than $4 \mathrm{mg} / \mathrm{ml}$ for 7 weeks in most of animals (Figure 2(a)). In some animal groups, glucose levels were elevated 1 week after STZ injection, decreased 2 weeks later, and then steadily increased up to 7-week time point. Glucose levels in $\mathrm{BeD}$ subgroup, unlike other subgroups, showed a tendency of decrease between 3 and 7 weeks.

It was reported that STZ treatment in vivo and in vitro evokes inflammatory responses as identified by the induction of inflammatory cytokines in the peripheral nerves [17]. Here we injected rats with STZ and, 1 week later, treated rats with herbal drugs for 2 weeks (3W-STZ group). STZ injection significantly increased TNF- $\alpha$ in the sciatic nerve, and the protein band intensity was decreased most dramatically by $\mathrm{BeD}$ treatment among the subgroup decoctions (Figure 2(b)). Immunofluorescence analysis showed that TNF- $\alpha$ signals in 3W-STZ animals were colocalized with NF-200 signals, indicating the presence of TNF- $\alpha$ in axons (Figure 2(c), arrows in the upper panel). TNF- $\alpha$ signals were also found in the regions which are in close contact with Hoechststained nuclei, suggesting the production of TNF- $\alpha$ from nonneuronal cells such as Schwann cells. (Figure 2(c), arrow in the lower panel). To examine the effects of subgroup decoctions on the neurite outgrowth, animals were injected with STZ, and DRGs were prepared from diabetes-induced rats 7 days later. Length of neurite was significantly shorter in DRG neurons from diabetic animals compared to those from untreated control (Figure 2(d)). Treatment of BeD significantly improved the neurite outgrowth while the other decoctions were little or slightly effective (Figures 2(d) and 2(e)). Taken together, these data suggest that a newly formulated $\mathrm{BeD}$ may protect the peripheral nerves from inflammation in STZ-diabetic animals and facilitate axonal growth.

Then, we investigated whether $\mathrm{BeD}$ was comparable to BGJTD in regulating neural responses in STZ-diabetic animals. Here in addition to $3 \mathrm{~W}$-STZ group, we prepared $6 \mathrm{~W}$ STZ animals in which BGJTD and BeD were administered for 2 weeks following a 4-week interval after STZ injection. In $3 \mathrm{~W}-\mathrm{STZ}$ animals, TNF- $\alpha$ production, which was induced by STZ injection, was slightly decreased by BGJTD and further decreased by $\mathrm{BeD}$ treatments (left panel in Figure 3(a)). Interestingly in $6 \mathrm{~W}-\mathrm{STZ}$ animals, TNF- $\alpha$ production was greatly suppressed by $\mathrm{BeD}$ (right panel in Figure 3(a)). To examine whether TNF- $\alpha$ production in the sciatic nerve was regulated at gene expression level, we analyzed mRNA expression by real-time PCR in the DRG at lumbar levels 4 and 5 where the cell bodies of sciatic sensory neurons are located. TNF- $\alpha$ mRNA levels in the DRG were significantly elevated in $3 \mathrm{~W}$ STZ rats compared to untreated control animals and then significantly downregulated by the administration of BGJTD and $\mathrm{BeD}$ (Figure 3(b)).
Elevated levels of blood glucose can induce signaling events such as the activation of MAP kinase pathway in the target cells by acting as osmotic and oxidative stressors [18]. We examined whether the treatment of BGJTD and BeD was involved in regulating phospho-Erk1/2 and p38 kinase levels in STZ-diabetic animals. Phospho-Erk1/2 level was elevated in the sciatic nerves in $3 \mathrm{~W}-\mathrm{STZ}$ animals compared with untreated control, and slightly upregulated by BeD treatment without showing statistical significance (Figure 4(a)). Histological examination of the sciatic nerves in $3 \mathrm{~W}-\mathrm{STZ}$ animal revealed that some, but not all, of induced phosphoErk1/2 signals were colocalized with NF-200-labeled axons (Figure 4(b)), suggesting that phospho-Erk1/2 signals were present in both axons and nonneuronal cells such as Schwann cells. It was further noted that some phospho-Erk1/2 signals were observed from the epineurial sheath (arrows in Figure 4(b)), which may contribute to phospho-Erk1/2 band intensity to some extent in the control and experimental groups in the western blot data of Figure 4(a). In 6W-STZ animals, phospho-Erk1/2 levels were slightly increased by STZ injection and then were highly upregulated by BGJTD and $\mathrm{BeD}$ treatments (Figure 4(c)). Phospho-Erk1/2 signals were mostly colocalized with S100 $\beta$-labeled Schwann cells in $\mathrm{BeD}$-treated STZ animals (Figure 4(d)). It was reported that Erk1/2 and p38 kinases play an opposing role in apoptosis of PC12 cells [19]. Having found that BGJTD and BeD strongly activated Erk1/2 in 6W-STZ animals, we investigated their regulatory effects on p38 production in the same animal groups. P38 was strongly induced by STZ and almost completely abolished by BGJTD and BeD treatments (Figure 4(e)), which was regulated in an opposite way to phospho-Erk1/2. In the sciatic nerve, some p38 signals were colocalized with NF-200-stained axons (arrow in Figure 4(f)) while others were seen around the Hoechst-stained nuclei (arrowhead in Figure 4(f)), indicating the production of p38 in both axons and nonneuronal cells such as Schwann cells.

Since we found that $\mathrm{BeD}$ showed the neuroprotective effects comparable to BGJTD in STZ-diabetic animals, we then asked whether individual constituents of $\mathrm{BeD}$ might have a similar activity. Treatment of $\mathrm{BeD}$ and AG significantly increased neurite outgrowth compared to saline control, showing a similar level of enhancement between them (Figure 5(a)). Then, treatment of MEK1/2 inhibitor PD98059 attenuated neurite growth most efficiently in neurons treated with AG drug. Phospho-Erk1/2 signals were increased in neurons and nonneuronal cells in culture by treatment of some drugs, showing strong signals in BeD- and AG-treated cells. It was noted that the cells bearing strong phospho-Erk1/2 signal revealed more intense processes in neurite outgrowth (Figure 5(b)). We also analyzed whether TNF- $\alpha$ production in DRG neurons was affected by treatment of $\mathrm{BeD}$ herbal components. As shown in Figure 5(c), TNF- $\alpha$ was found only in neuronal cell body, and its level was relatively lower in BeD- and AG-treated cells. Taken together, AG, a herbal component of $\mathrm{BeD}$, may induce neurite outgrowth efficiently by regulating Erk1/2 activity and TNF- $\alpha$ production in DRG neurons.

We then examined the effects of BGJTD and BeD on the thermal sensitivity in the hind limbs in mice. The latency 


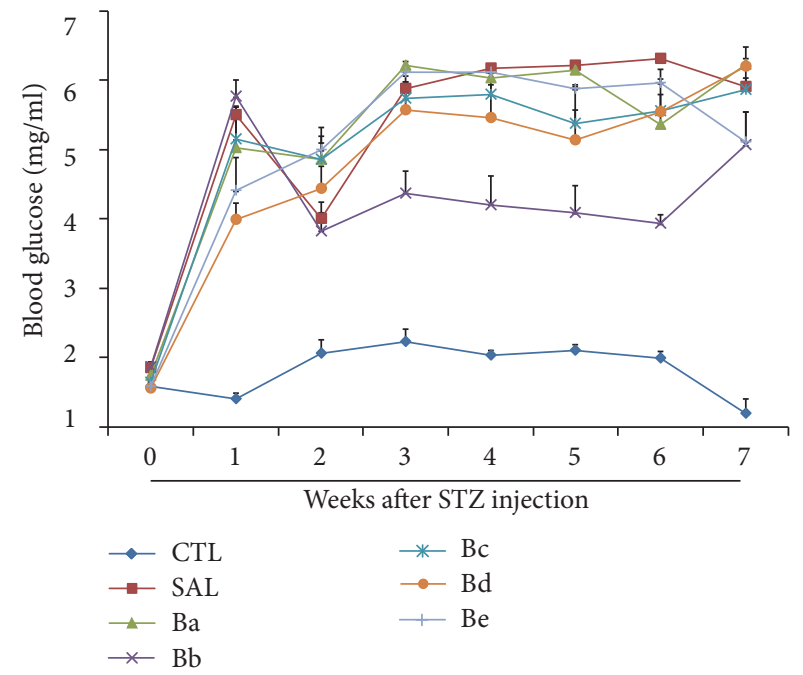

(a)
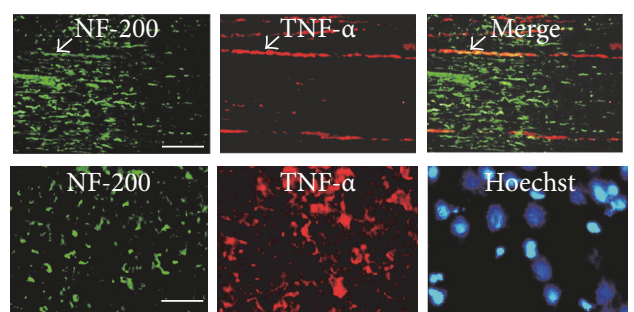

(c)
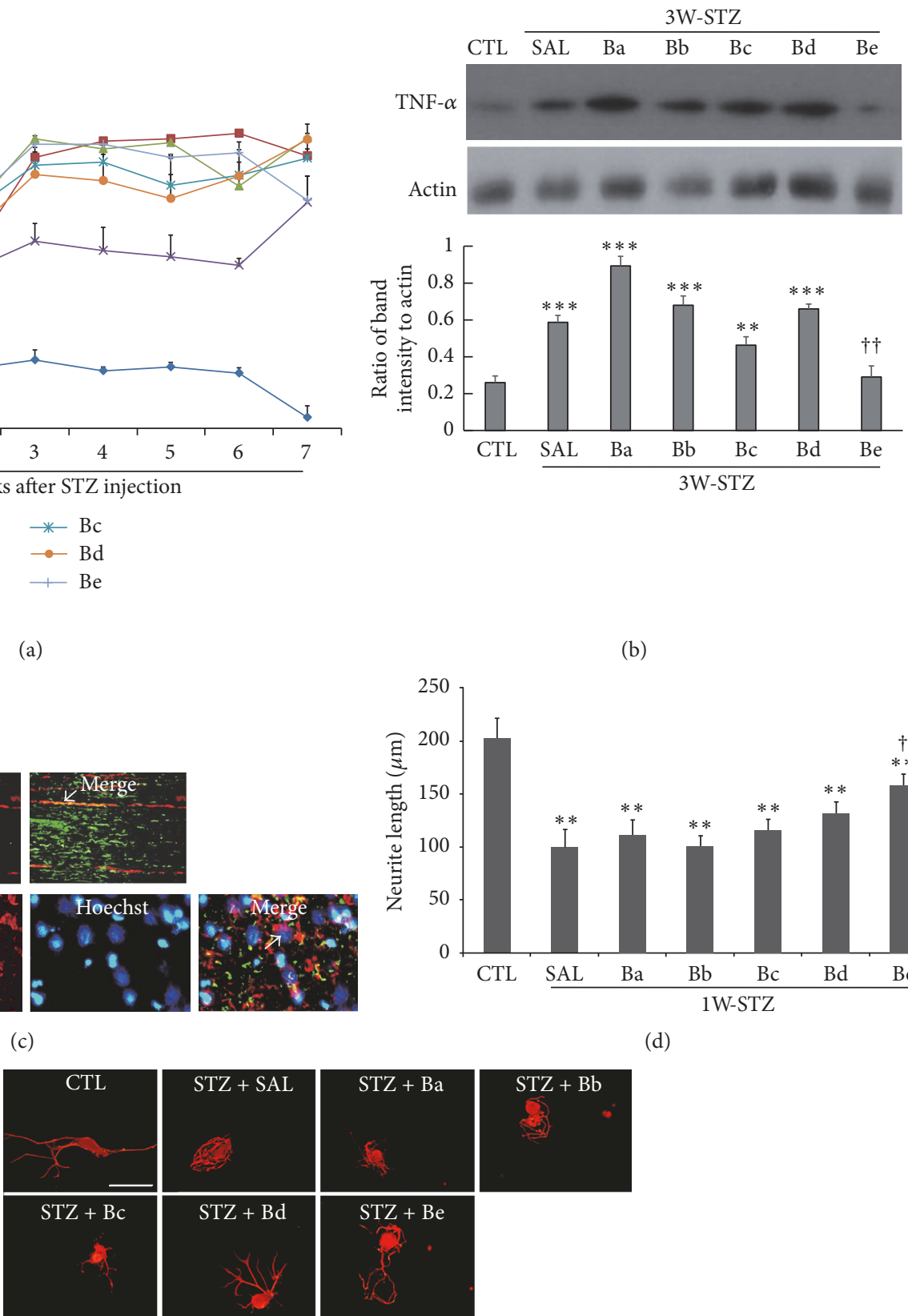

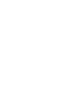

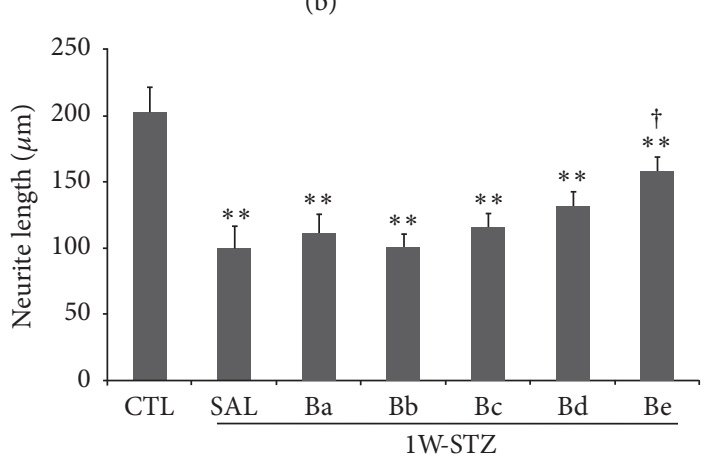

(d) 


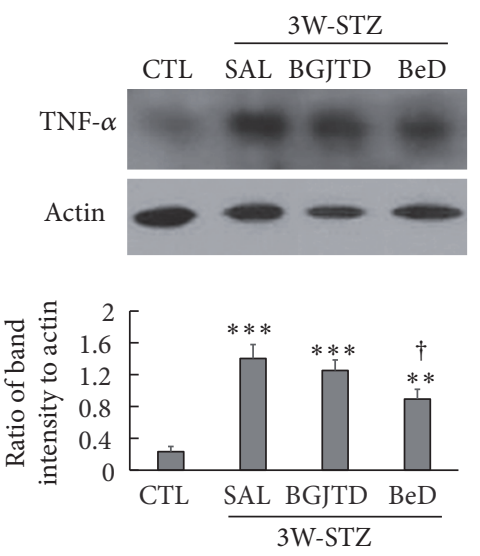

(a)

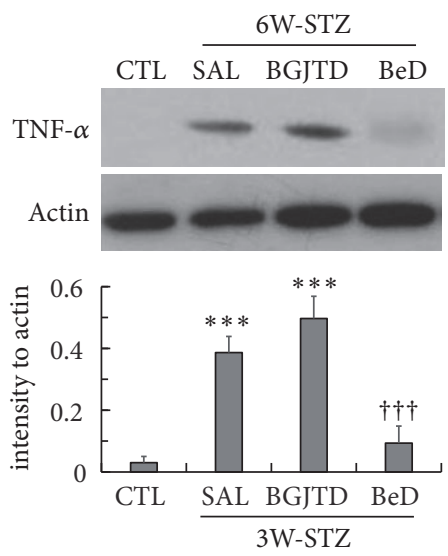

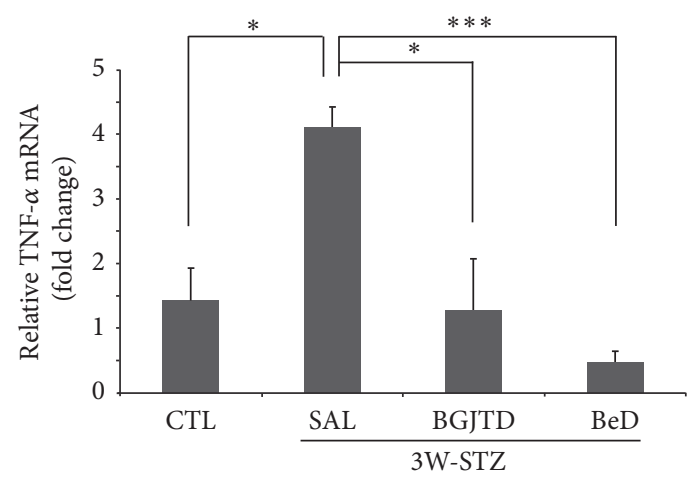

(b)

FIGURE 3: Comparison of TNF- $\alpha$ levels in the sciatic nerve of STZ-diabetic animals after herbal drug treatments. (a) Western blot analysis of TNF- $\alpha$ in the sciatic nerves from 3W-STZ and 6W-STZ animal groups. Animals were injected with STZ and, 1 week and 4 weeks later, subjected to daily administration of herbal drugs for 2 weeks (labeled 3W-STZ and 6W-STZ, resp.). Images in the upper panel show the representatives from 3 independent experiments, and quantitation of protein band intensity relative to actin control is shown in the lower panel. ${ }^{* *} p<0.01,{ }^{* * *} p<0.001$ versus untreated control (CTL); ${ }^{\dagger} p<0.05,{ }^{\dagger \dagger \dagger} p<0.001$ versus saline control. (b) Real-time PCR for the comparison of TNF- $\alpha$ mRNA expression in the DRG neurons among experimental groups. ${ }^{*} p<0.05,{ }^{* * *} p<0.001$. Number of independent experiments $=4$.

time for withdrawal responses on the hot plate was greatly increased by STZ injection and was significantly reduced by BGJTD and BeD treatments (Figure 6(a)). A withdrawal frequency from the hot plate was significantly decreased in STZ-treated animals compared to untreated control animals. Treatment of BGJTD and BeD failed to show significant changes in withdrawal frequency in STZ-treated group (Figure 6(b)).

\section{Discussion}

BGJTD formulation has been developed and used therapeutically in the traditional Korean medicine to alleviate neuropathy associated with diabetes [7]. To understand the biological basis on its efficacy, previously we studied the neural responses in animals given taxol and crush injury to the sciatic nerves and found the protective activity of BGJTD in the injured nerves [8]. Here using biochemical, histological, and behavioral methods, we found that BGJTD, together with an additionally formulated decoction selected from BGJTD, was effective in protecting the peripheral nerves from neuropathic insults in STZ-diabetic animals.

BGJTD is a mixture of herbal drugs composed of 18 different components. Measurement of neurite outgrowth in cultured neurons is a convenient way to examine the effects of candidate drugs on the regenerative responses of neurons given injury [20]. Previous studies also showed that the intrinsic capacity of axonal regeneration after nerve injury and neurite outgrowth are retarded in STZ-induced diabetic animals $[21,22]$, which may reflect the pathological impairment of axonal function such as increases in inhibitory factors of axonal regeneration and downregulation of proinflammatory cytokines $[23,24]$. Thus, as an initial step to select active herbal components, we treated DRG neurons with individual herbal drugs and selected the most active component from each subgroup. Drugs such as PG, AG, PL, and CG induced the neurite outgrowth most efficiently from each subgroup and thus used for constituting a new formula Be. It was previously reported that levels of TNF- $\alpha$ were elevated in the serum, brain tissue, and peripheral nerves in STZ-diabetic animals $[25,26]$, and its level was further upregulated in the serum by LPS injection [27]. In the present study, TNF- $\alpha$ was induced in the sciatic nerve in rats 3 weeks after STZ injection. Strong TNF- $\alpha$ signals were colocalized with NF-200-labeled nerve fibers, and additional signals were observed in the surrounding nonneuronal cells such as Schwann cells as reported previously [28]. Examination of subgroup decoctions on the regulation of inflammatory responses in STZ-diabetic animals revealed that $\mathrm{BeD}$ was most effective in downregulating TNF- $\alpha$ levels in the sciatic nerve of STZ-diabetic animals. Treatment of BGJTD and BeD similarly attenuated TNF- $\alpha$ mRNA expression in the DRG, suggesting that BGJTD and its active components may be involved in regulating TNF- $\alpha$ production in neuronal cells and Schwann cells at gene expression level.

Since $\mathrm{BeD}$ was the collection of active herbal components inducing the neurite outgrowth, we investigated whether BeD was superior to other subgroup decoctions in inducing neurite outgrowth. Neurite outgrowth was largely retarded in DRG neurons which had been prepared from STZ-diabetic rats compared control animals. These data suggest that, on the contrary of the potential effects of preconditioning injury for the enhancement of neurite growth [29], hyperglycemic environment by STZ treatment may suppress the gene expression in DRG neurons leading to attenuate axonal growth. BeD facilitated neurite outgrowth most efficiently, implying that the herbal constituents of $\mathrm{BeD}$ may protect DRG neurons from the neuropathic insults by STZ. 


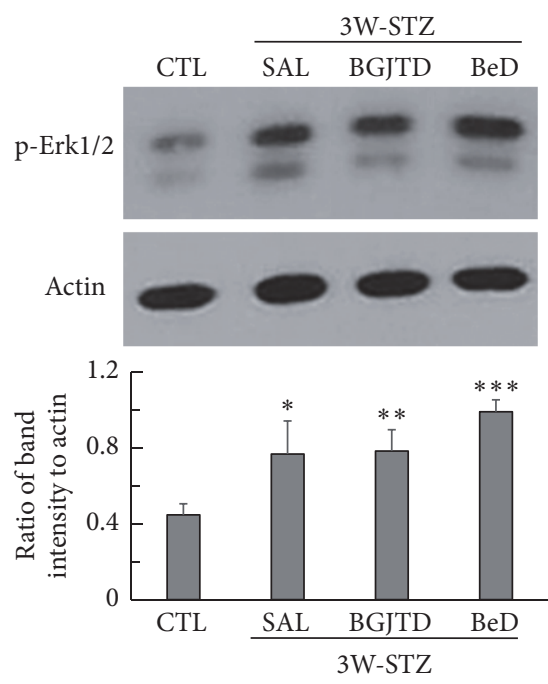

(a)
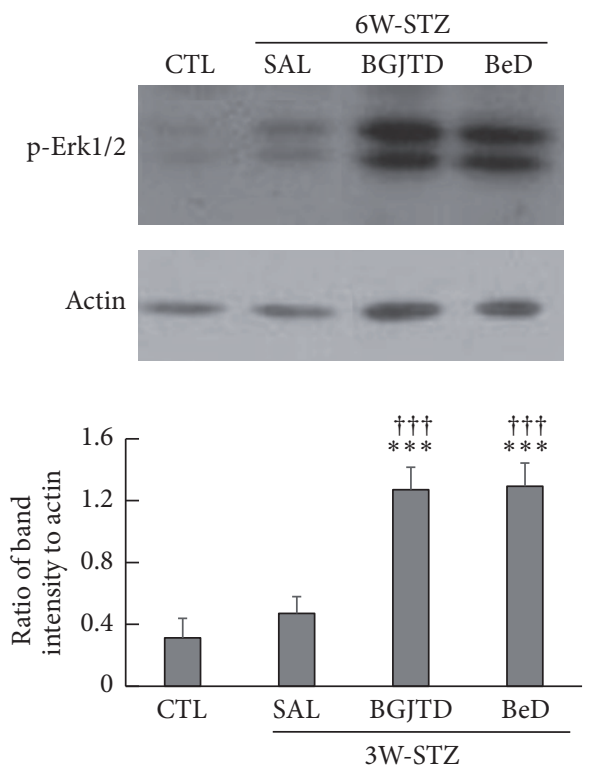

(c)
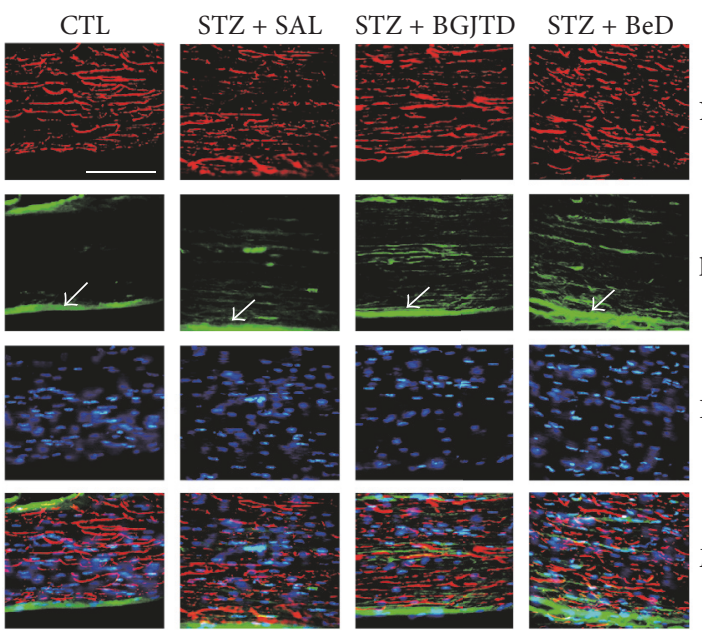

NF-200
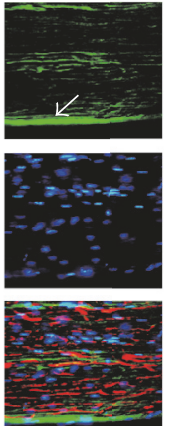

(b)
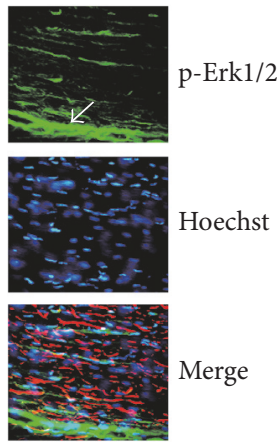
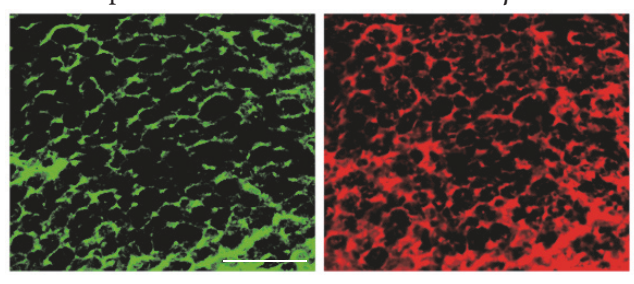

(d)

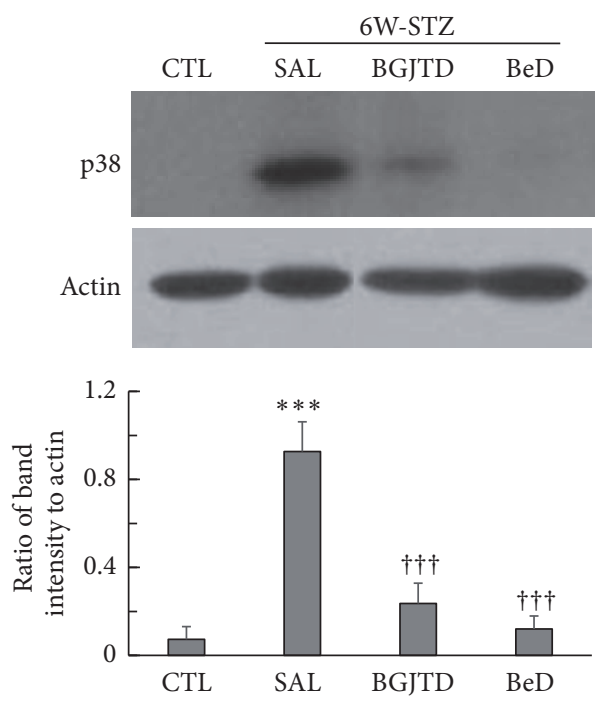

(e)

Figure 4: Continued. 

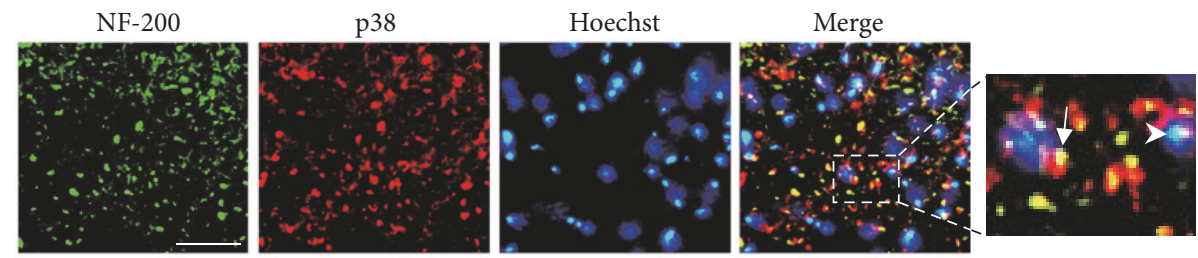

(f)

FIGURE 4: Induction pattern of phospho-Erk1/2 and p38 proteins in the sciatic nerve of STZ-diabetic animals after herbal drug treatments. Animals were injected with STZ and, 1 week and 4 weeks later, subjected to daily administration of herbal drugs for 2 weeks (labeled $3 \mathrm{~W}-\mathrm{STZ}$ and 6W-STZ respectively). (a) Western blot analysis of phospho-Erk1/2 in the sciatic nerve from $3 \mathrm{~W}-\mathrm{STZ}$ animal group. (b) Immunofluorescence images showing phospho-Erk1/2 signals in NF-200-stained sciatic nerve sections, which were prepared from BeDtreated 3W-STZ animals. Phospho-ERK1/2 signals in the epineurial sheath are indicated by arrows. (c) Western blot analysis of phosphoErk1/2 in the sciatic nerve from 6W-STZ animal group. (d) Immunofluorescence images showing phospho-Erk1/2 and S100 $\beta$ signals in the transverse nerve section, which were prepared from BeD-treated 6W-STZ animals. (e) Western blot analysis of p38 in the sciatic nerve from $6 \mathrm{~W}-\mathrm{STZ}$ animal group. (f) Immunofluorescence images showing p38 and NF-200 signals in the transverse nerve section, which were prepared from saline-treated 6W-STZ animals. Western blotting images in (a), (c), and (e) are the representatives from 3 independent experiments (upper panels), and quantitation of protein band intensity relative to actin control is shown in the lower panels. ${ }^{*} p<0.01,{ }^{* *} p<0.01$, ${ }^{* * *} p<0.001$ versus untreated control (CTL); ${ }^{\dagger \dagger} p<0.001$ versus saline control. Scale bars in (b), (d), and (f) $=100 \mu \mathrm{m}$.

Having confirmed that BeD regulated inflammatory reactions in the sciatic nerves and promoted neurite outgrowth, we explored its effects on neural tissues in vivo. Induction levels of phospho-Erk1/2, which was measured as an indicator of activation of cells in the sciatic nerve, were different between $3 \mathrm{~W}-\mathrm{STZ}$ and 6-W STZ animal groups. Then, phospho-Erk1/2 was upregulated by $\mathrm{BGJTD}$ and $\mathrm{BeD}$ treatment in both groups, suggesting the activation for neural pathway for survival. In the sciatic nerves, some, but not all, of phosphoErk1/2 signals were observed in axons. Phospho-Erk1/2 can be induced by neurotrophic signaling in the peripheral nerves given injury and retrogradely transported into the cell body where it is involved in target gene expression [30-32]. Here, we speculate that axonal phospho-Erk1/2 signals, which were initially induced by osmotic stress in STZ-diabetic animals, were further upregulated by $\mathrm{BGJTD}$ and $\mathrm{BeD}$ to activate intracellular signaling pathway in DRG neurons. In addition, phospho-Erk1/2 signals found in Schwann cells may play a role in regulating neural activity locally. In contrast to neuronal survival by Erk1/2 activation, activation of p38 and JNK pathways was known to activate apoptotic pathway in PC12 cell [19]. Indeed, our data showed that p38 protein, which was increased by STZ injection, was dramatically decreased by BGJTD and BeD treatments. We also noted that phospho-Erk1/2 and p38 signals were observed in Schwann cells in the sciatic nerve in STZ-diabetic animals. Although our results do not provide direct evidence on the regulation of these proteins in Schwann cells, it is highly likely that BGJTD and $\mathrm{BeD}$ act on Schwann cells, considering that Schwann cell interaction with axons is critical for the recovery from neuropathic pain and nerve injury $[33,34]$. Phospho-Erk1/2 and p38 may respond to BGJTD and BeD treatments in an opposite way in the sciatic nerve axons and Schwann cells so as to protect neurons and Schwann cells from the hyperglycemic stress in diabetic animals. Future studies on the induction pattern of neurotrophic factors and inflammatory cytokines may provide insights into mechanistic basis on the signaling event mediated by BGJTD and BeD in STZ-diabetic animals.
Analysis of behavioral response showed that treatments of $\mathrm{BDJTD}$ and $\mathrm{BeD}$ reduced the latency time responding to thermal stimulation while both drugs failed to change withdrawal frequency. Decreased latency to heat may indicate the improvement of reflexive behavior to thermal stimulation and associated pain [35]. Whether these behavioral changes are linked to biochemical responses in the sciatic nerves remains to be explored.

\section{Conclusion}

Our study shows that $\mathrm{BGJTD}$ and $\mathrm{BeD}$ improved neural responses in the sciatic nerves from neuropathic insults in STZ-diabetic animals. BGJTD is a herbal formulation which has been prescribed for the treatment of diabetic neuropathic pain in clinical practice in Korea [7]. A newly formulated $\mathrm{BeD}$ is composed of four herbal components and they may be involved in elevating erythropoietic activity, tonifying qi and blood flow into some stagnant body parts, and replenishing yin energy while subduing yang, according to traditional medicinal theory. Consequently, augmented vital energy may alleviate pain which is caused by stagnation of qi and blood flow. Ginsenoside Rb1 isolated from PG and albiflorin and paeoniflorin both from PL have been identified as the chemical ingredients of $\mathrm{BeD}$ [8]. Growing body of evidence indicates that $\mathrm{Rb} 1$ has a protective effect on neural tissues related to inflammatory and degenerative diseases [36]. The isomers albiflorin and paeoniflorin were reported to have anti-inflammatory activity and regulate neuropathic pain in spinal neurons [37, 38]. Interestingly, a herbal recipe called Tang Luo Ning, which contains both albiflorin and paeoniflorin among others, was shown to attenuate oxidative stress in STZ-induced diabetic rats [39]. Finally, our data demonstrate that treatment of AG into the DRG neurons from STZ-diabetic animals increases neurite outgrowth and regulates the production of phospho-Erk1/2 and TNF- $\alpha$. Therefore, it is tempting to design pharmacological studies exploring the signaling pathways mediated 


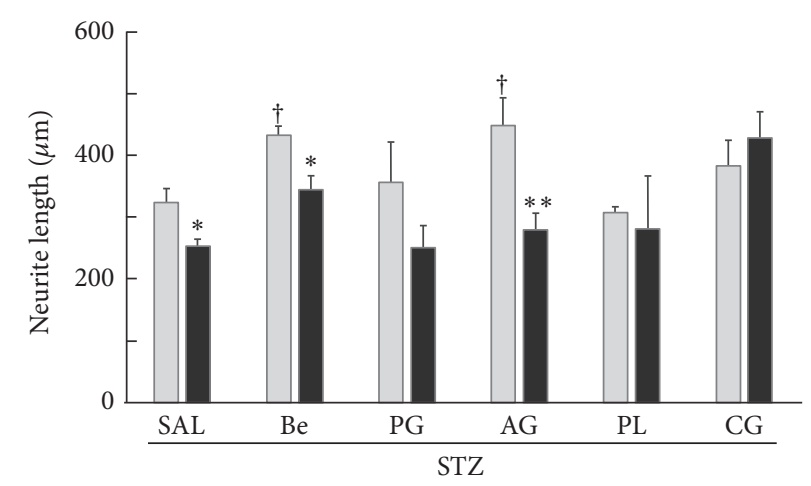

$\square$ Vehicle

- PD98059
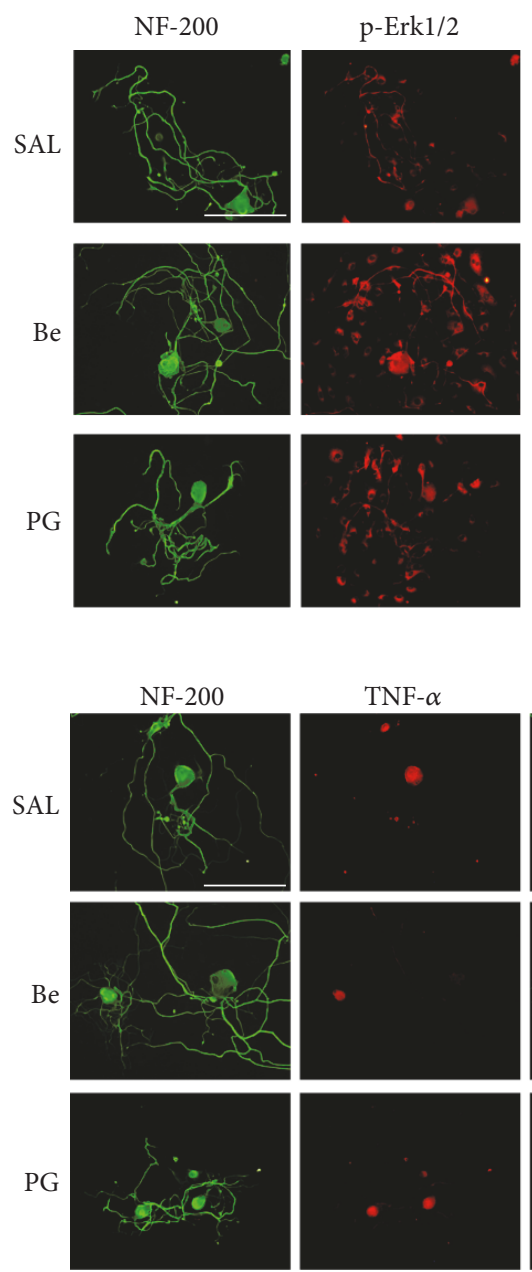

(a)
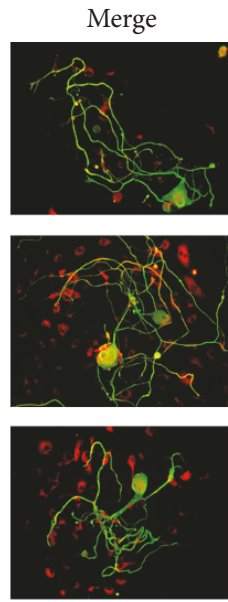

(b)
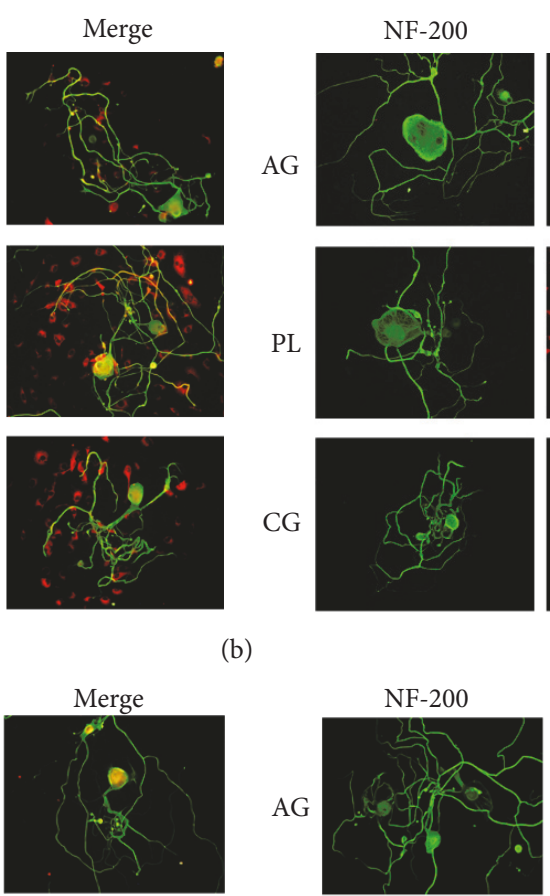

(b)
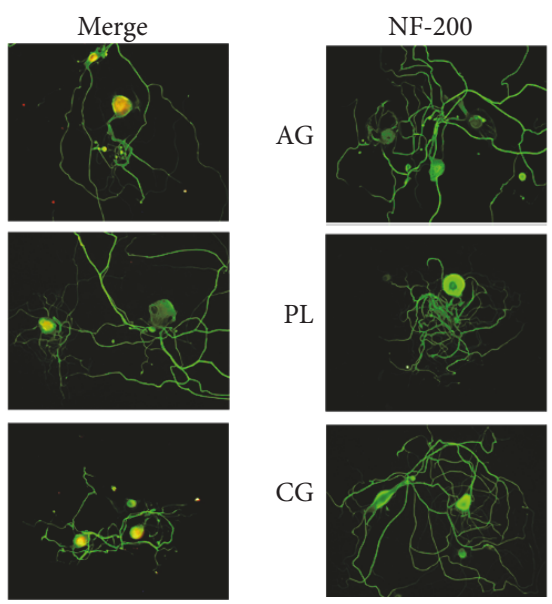

PL

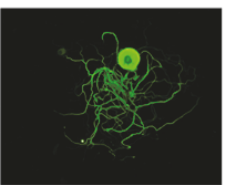

CG

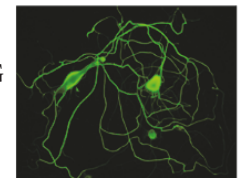

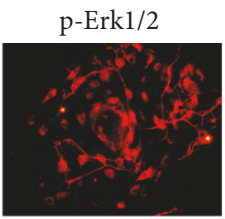
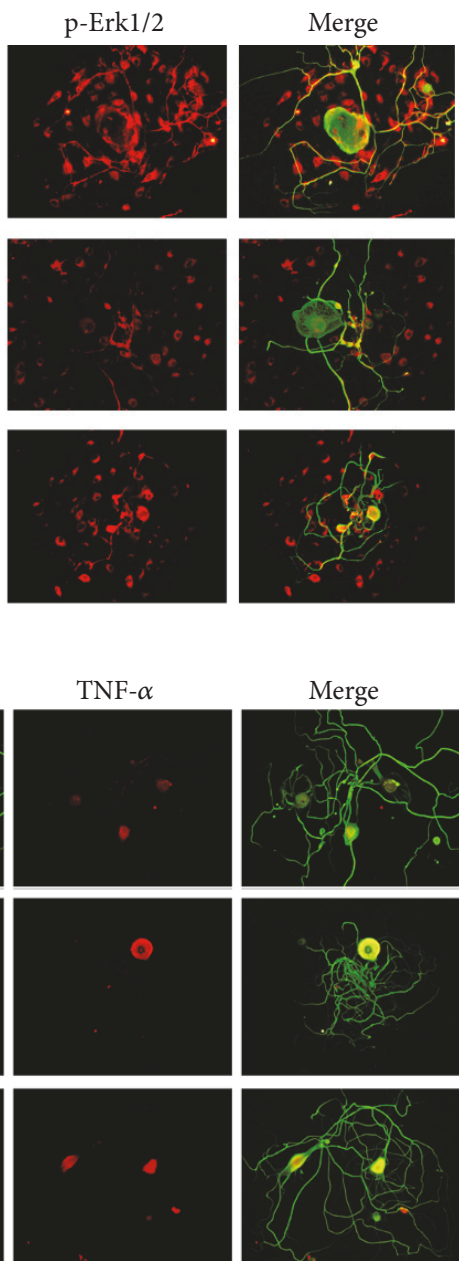

(c)

Figure 5: Effects of BeD herbal components on neurite outgrowth and the production of phospho-Erk1/2 and TNF- $\alpha$ in DRG neurons. (a) Comparison of neurite outgrowth in DRG neurons after treatment of herbal drugs. Cells were treated with PD98059 (10 $\mu$ M) or DMSO vehicle for $4 \mathrm{~h}$ before the addition of herbal drugs and cultured for $48 \mathrm{~h} .{ }^{*} p<0.05,{ }^{* *} p<0.01$ versus corresponding DMSO vehicle-treated groups; ${ }^{\dagger} p<0.05$ versus saline control. Number of independent experiments $=4$. (b). Immunofluorescence views of NF-200 and phospho-Erk1/2 signals in DRG neurons. (c) Immunofluorescence views of NF-200 and TNF- $\alpha$ signals in DRG neurons. In (a)-(c), DRG was prepared from STZ-diabetic rats, and cultured cells were treated with herbal drugs $(0.5 \mathrm{mg} / \mathrm{ml})$ for $48 \mathrm{~h}$ before cell harvest for immunofluorescence staining. Scale bars in (b) and (c) $=100 \mu \mathrm{m}$. 


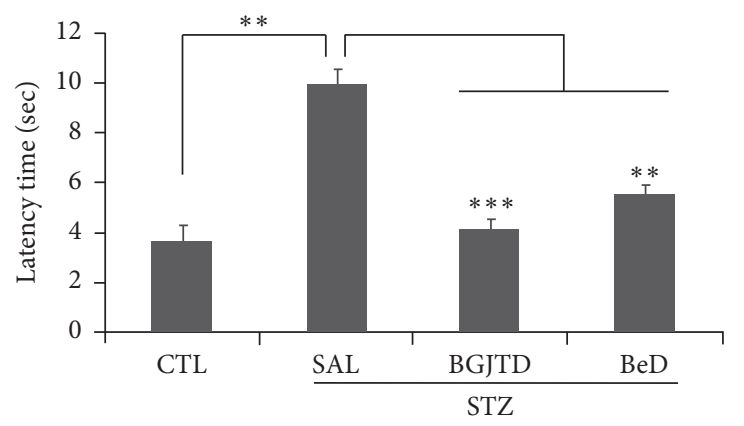

(a)

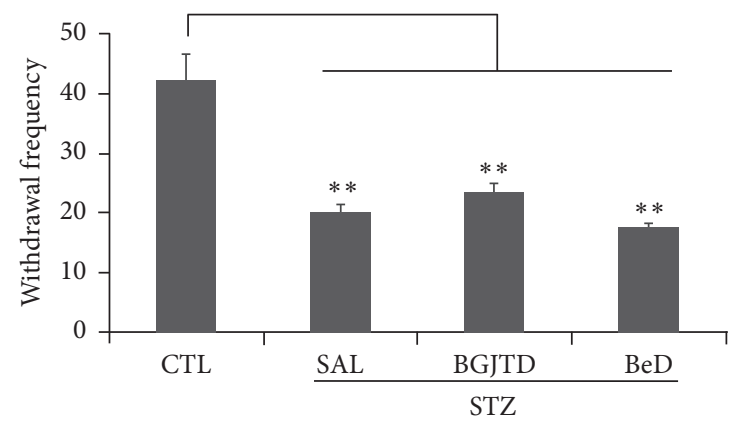

(b)

FIGURE 6: Effects of BGJTD and BeD on the heat sensitivity. STZ-diabetic mice were orally given BGJTD and BeD for 2 weeks and were subjected to the measurement of the lifting frequency of the hind paws (a) and the latency period after placing on the hot plate (b). ${ }^{* *} p<0.01$; ${ }^{* * *} p<0.001$. Number of animals $=4$.

by chemical ingredients and herbal components of $\mathrm{BeD}$. In conclusion, the present results suggest that the experimental reductionism may be applied to explore the biological basis on the efficacies of herbal drugs or decoctions.

\section{Conflicts of Interest}

The authors declare no conflicts of interest.

\section{Authors' Contributions}

Uk Namgung and Chung Sik Cho conceived and designed the experiments; Ki-Joong Kim, Uk Namgung, and Chung Sik Cho performed the experiments, interpreted the data, and wrote the main manuscript; all of the authors read and approved the final manuscript.

\section{Acknowledgments}

This research was supported by a grant of the Korea Health Technology R\&D Project through the Korea Health Industry Development Institute (KHIDI), funded by the Ministry of Health \& Welfare, Republic of Korea (Grant no. HI14C0963) and by the Basic Science Research Programs through the National Research Foundation of Korea (NRF) funded by the Ministry of Education (NRF-201602800001). The authors thank I.A. Chang and B.G. Jo for technical assistance.

\section{References}

[1] A. Menke, S. Casagrande, L. Geiss, and C. C. Cowie, "Prevalence of and trends in diabetes among adults in the United States, 1988-2012," The Journal of the American Medical Association, vol. 314, no. 10, pp. 1021-1029, 2015.

[2] G. Chen, R. Wang, H. Chen, L. Wu, R.-S. Ge, and Y. Wang, "Gossypol ameliorates liver fibrosis in diabetic rats induced by high-fat diet and streptozocin," Life Sciences, vol. 149, pp. 58-64, 2016.

[3] T. Szkudelsk, "The mechanism of alloxan and streptozotocin action in B cells of the rat pancreas," Physiological Research, vol. 50, no. 6, pp. 537-546, 2001.
[4] L. Daulhac, C. Mallet, C. Courteix et al., "Diabetes-induced mechanical hyperalgesia involves spinal mitogen-activated protein kinase activation in neurons and microglia via N-methylD-aspartate- dependent mechanisms," Molecular Pharmacology, vol. 70, no. 4, pp. 1246-1254, 2006.

[5] R. Wodarski, A. K. Clark, J. Grist, F. Marchand, and M. Malcangio, "Gabapentin reverses microglial activation in the spinal cord of streptozotocin-induced diabetic rats," European Journal of Pain, vol. 13, no. 8, pp. 807-811, 2009.

[6] H. Yamamoto, Y. Shimoshige, T. Yamaji, N. Murai, T. Aoki, and N. Matsuoka, "Pharmacological characterization of standard analgesics on mechanical allodynia in streptozotocin-induced diabetic rats," Neuropharmacology, vol. 57, no. 4, pp. 403-408, 2009.

[7] J. M. Kim, C. S. Cho, and C. J. Kim, "Clinical study of 8 diabetic patients with paresthesia," Korean Journal of Oriental Internal Medicine, vol. 31, pp. 184-191, 2010.

[8] S. H. Ahn, I. A. Chang, K.-J. Kim, C.-J. Kim, U. Namgung, and C.-S. Cho, "Bogijetong decoction and its active herbal components protect the peripheral nerve from damage caused by taxol or nerve crush," BMC Complementary and Alternative Medicine, vol. 16, no. 1, article no. 402, 2016.

[9] L. Jong bin, K. C. Jung, and C. C. Sik, "Effects of bogijetong-tang on diabetic-peripheral neuropathy induced by streptozotocin in the mouse," Journal of Korean Medicine, vol. 34, no. 3, pp. 126142, 2013.

[10] S. Sancheti and S.-Y. Seo, "Antidiabetic and antiacetylcholinesterase effects of ethyl acetate fraction of Chaenomeles sinensis (Thouin) Koehne fruits in streptozotocin-induced diabetic rats," Experimental and Toxicologic Pathology, vol. 65, no. 1-2, pp. 55-60, 2013.

[11] Y. Zhong, X. Zhang, X. Cai, K. Wang, Y. Chen, and Y. Deng, "Puerarin attenuated early diabetic kidney injury through down-regulation of matrix metalloproteinase 9 in streptozotocin-induced diabetic rats," PLoS ONE, vol. 9, no. 1, Article ID e85690, 2014.

[12] B.-V. Lim, M.-C. Shin, M.-H. Jang et al., "Ginseng radix increases cell proliferation in dentate gyrus of rats with streptozotocin-induced diabetes," Biological and Pharmaceutical Bulletin, vol. 25, no. 12, pp. 1550-1554, 2002.

[13] S. Sen, S. Chen, B. Feng, Y. Wu, E. Lui, and S. Chakrabarti, "Preventive effects of North American ginseng (Panax quinquefolium) on diabetic nephropathy," Phytomedicine, vol. 19, no. 6, pp. 494-505, 2012. 
[14] Y. J. Hong, N. Kim, K. Lee et al., "Korean red ginseng (Panax ginseng) ameliorates type 1 diabetes and restores immune cell compartments," Journal of Ethnopharmacology, vol. 144, no. 2, pp. 225-233, 2012.

[15] H.-D. Lim, M.-H. Kim, C.-Y. Lee, and U. Namgung, "Antiinflammatory effects of acupuncture stimulation via the vagus nerve," PLoS ONE, vol. 11, no. 3, Article ID e0151882, 2016.

[16] I. S. Han, T. B. Seo, K.-H. Kim, J.-H. Yoon, S.-J. Yoon, and U. Namgung, "Cdc2-mediated Schwann cell migration during peripheral nerve regeneration," Journal of Cell Science, vol. 120, no. 2, pp. 246-255, 2007.

[17] M. Zychowska, E. Rojewska, B. Przewlocka, and J. Mika, "Mechanisms and pharmacology of diabetic neuropathy-experimental and clinical studies," Pharmacological Reports, vol. 65, no. 6, pp. 1601-1610, 2013.

[18] C. Tohda, T. Kuboyama, and K. Komatsu, "Search for natural products related to regeneration of the neuronal network," NeuroSignals, vol. 14, no. 1-2, pp. 34-45, 2005.

[19] J. M. Kennedy and D. W. Zochodne, "The regenerative deficit of peripheral nerves in experimental diabetes: its extent, timing and possible mechanisms," Brain, vol. 123, no. 10, pp. 2118-2129, 2000.

[20] Y. Sakaue, M. Sanada, T. Sasaki, A. Kashiwagi, and H. Yasuda, "Amelioration of retarded neurite outgrowth of dorsal root ganglion neurons by overexpression of PKC $\delta$ in diabetic rats," NeuroReport, vol. 14, no. 3, pp. 431-436, 2003.

[21] K. Sango, H. Mizukami, H. Horie, and S. Yagihashi, "Impaired axonal regeneration in diabetes. perspective on the underlying mechanism from in vivo and in vitro experimental studies," Frontiers in Endocrinology, vol. 8, no. 12, pp. 1-8, 2017.

[22] T. Habash, A. Saleh, S. K. Roy Chowdhury, D. R. Smith, and P. Fernyhough, "The proinflammatory cytokine, interleukin$17 \mathrm{~A}$, augments mitochondrial function and neurite outgrowth of cultured adult sensory neurons derived from normal and diabetic rats," Experimental Neurology, vol. 273, pp. 177-189, 2015.

[23] V. Solmaz, B. P. Çınar, G. Yiğittürk, T. Çavuşoğlu, D. Taşkıran, and O. Erbaş, "Exenatide reduces TNF- $\alpha$ expression and improves hippocampal neuron numbers and memory in streptozotocin treated rats," European Journal of Pharmacology, vol. 765, pp. 482-487, 2015.

[24] X. Qiang, J. Satoh, M. Sagara et al., "Gliclazide inhibits diabetic neuropathy irrespective of blood glucose levels in streptozotocin-induced diabetic rats," Metabolism: Clinical and Experimental, vol. 47, no. 8, pp. 977-981, 1998.

[25] A. Kumar, G. Negi, and S. S. Sharma, "Suppression of NF- $\kappa$ B and NF- $\kappa$ B regulated oxidative stress and neuroinflammation by BAY 11-7082 ( $\mathrm{\kappa} \mathrm{B}$ phosphorylation inhibitor) in experimental diabetic neuropathy," Biochimie, vol. 94, no. 5, pp. 1158-1165, 2012.

[26] X. Qiang, J. Satoh, M. Sagara et al., "Inhibitory effect of troglitazone on diabetic neuropathy in streptozotocin-induced diabetic rats," Diabetologia, vol. 41, no. 11, pp. 1321-1326, 1998.

[27] Y. Qin, M. Hua, Y. Duan et al., "TNF- $\alpha$ expression in schwann cells is induced by LPS and NF- $\kappa$ B-dependent pathways," Neurochemical Research, vol. 37, no. 4, pp. 722-731, 2012.

[28] D. S. Smith and J. H. P. Skene, "A transcription-dependent switch controls competence of adult neurons for distinct modes of axon growth," Journal of Neuroscience, vol. 17, no. 2, pp. 646658, 1997.
[29] R. A. Segal and M. E. Greenberg, "Intracellular signaling pathways activated by neurotrophic factors," Annual Review of Neuroscience, vol. 19, pp. 463-489, 1996.

[30] S. Hanz and M. Fainzilber, "Retrograde signaling in injured nerve-the axon reaction revisited," Journal of Neurochemistry, vol. 99, no. 1, pp. 13-19, 2006.

[31] T. B. Seo, M.-J. Oh, B.-G. You et al., "ERK1/2-mediated schwann cell proliferation in the regenerating sciatic nerve by treadmill training," Journal of Neurotrauma, vol. 26, no. 10, pp. 1733-1744, 2009.

[32] Z. Xia, M. Dickens, J. Raingeaud, R. J. Davis, and M. E. Greenberg, "Opposing effects of ERK and JNK-p38 MAP kinases on apoptosis," Science, vol. 270, no. 5240, pp. 1326-1331, 1995.

[33] J. Scholz and C. J. Woolf, "The neuropathic pain triad: neurons, immune cells and glia," Nature Neuroscience, vol. 10, no. 11, pp. 1361-1368, 2007.

[34] U. Namgung, "The role of schwann cell-axon interaction in peripheral nerve regeneration," Cells Tissues Organs, vol. 200, no. 1, pp. 6-12, 2014.

[35] M. Baliki, O. Calvo, D. R. Chialvo, and A. V. Apkarian, "Spared nerve injury rats exhibit thermal hyperalgesia on an automated operant dynamic thermal escape task," Molecular Pain, vol. 1, article no. 18, 2005.

[36] T. Ahmed, S. H. Raza, A. Maryam et al., "Ginsenoside Rb1 as a neuroprotective agent: a review," Brain Research Bulletin, vol. 125, pp. 30-43, 2016.

[37] J. Zhou, L. Wang, J. Wang et al., "Paeoniflorin and albiflorin attenuate neuropathic pain via MAPK pathway in chronic constriction injury rats," Evidence-based Complementary and Alternative Medicine, vol. 2016, Article ID 8082753, 11 pages, 2016.

[38] Q.-S. Wang, T. Gao, Y.-L. Cui, L.-N. Gao, and H.-L. Jiang, "Comparative studies of paeoniflorin and albiflorin from Paeonia lactiflora on anti-inflammatory activities," Pharmaceutical Biology, vol. 52, no. 9, pp. 1189-1195, 2014.

[39] X. Yang, W. Yao, Q. Li et al., "Mechanism of Tang Luo Ning effect on attenuating of oxidative stress in sciatic nerve of STZinduced diabetic rats," Journal of Ethnopharmacology, vol. 174, pp. 1-10, 2015. 


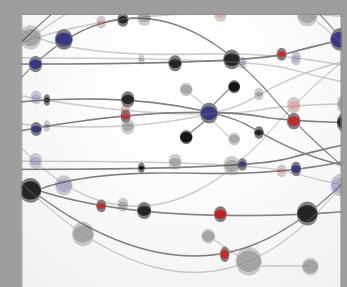

The Scientific World Journal
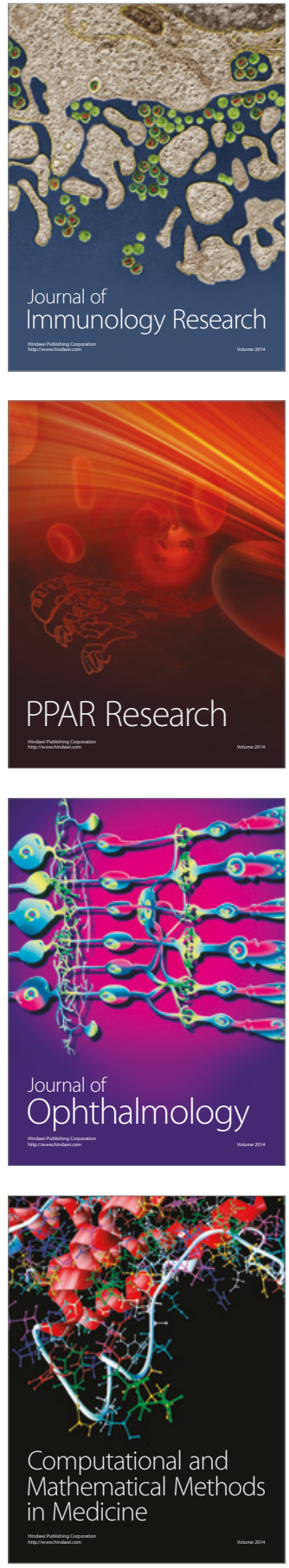

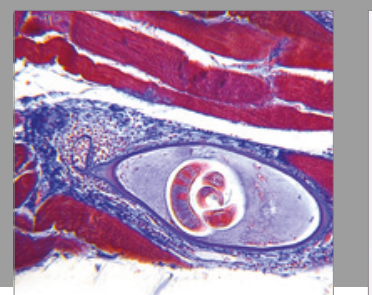

Gastroenterology Research and Practice
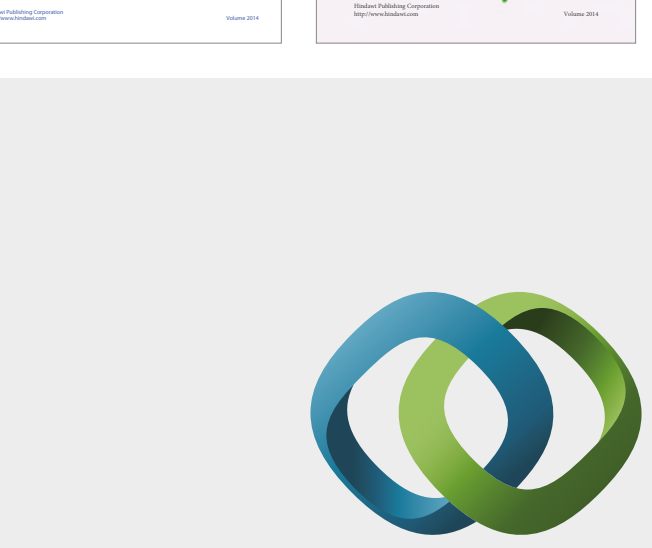

\section{Hindawi}

Submit your manuscripts at

https://www.hindawi.com
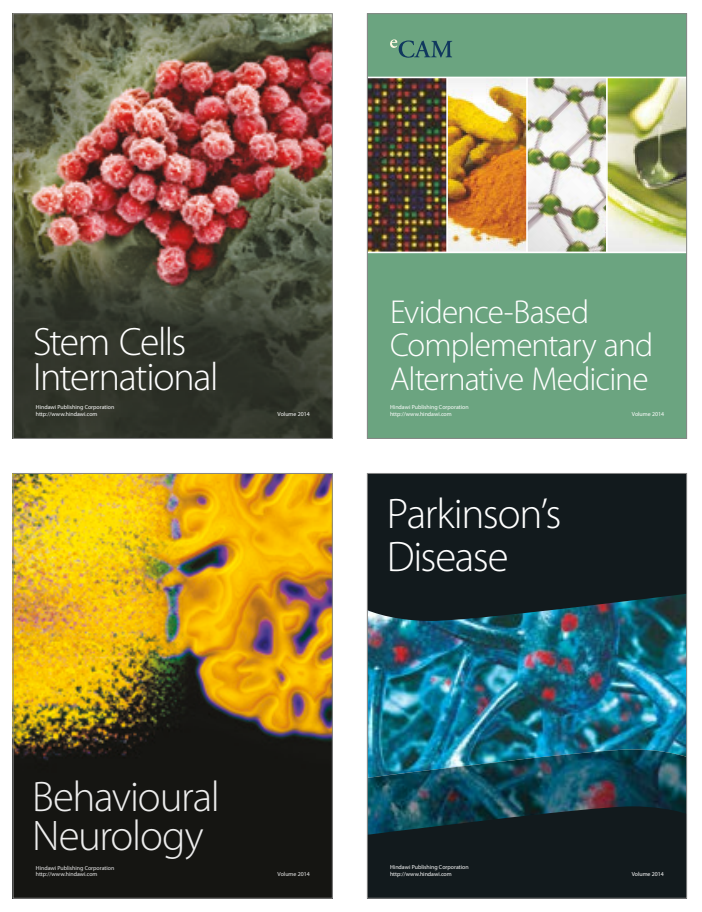
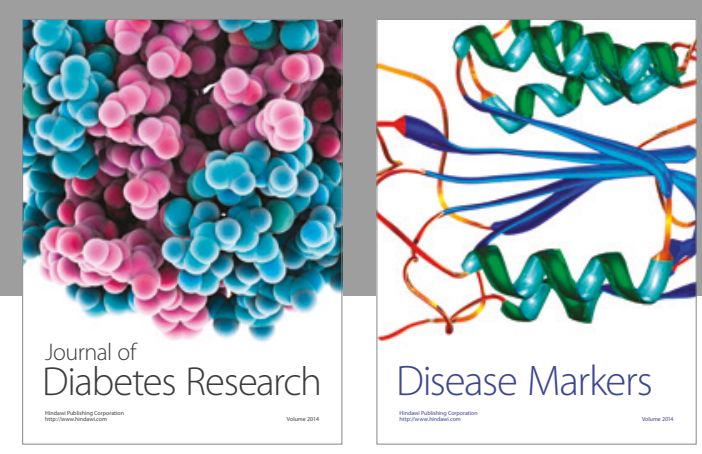

Disease Markers
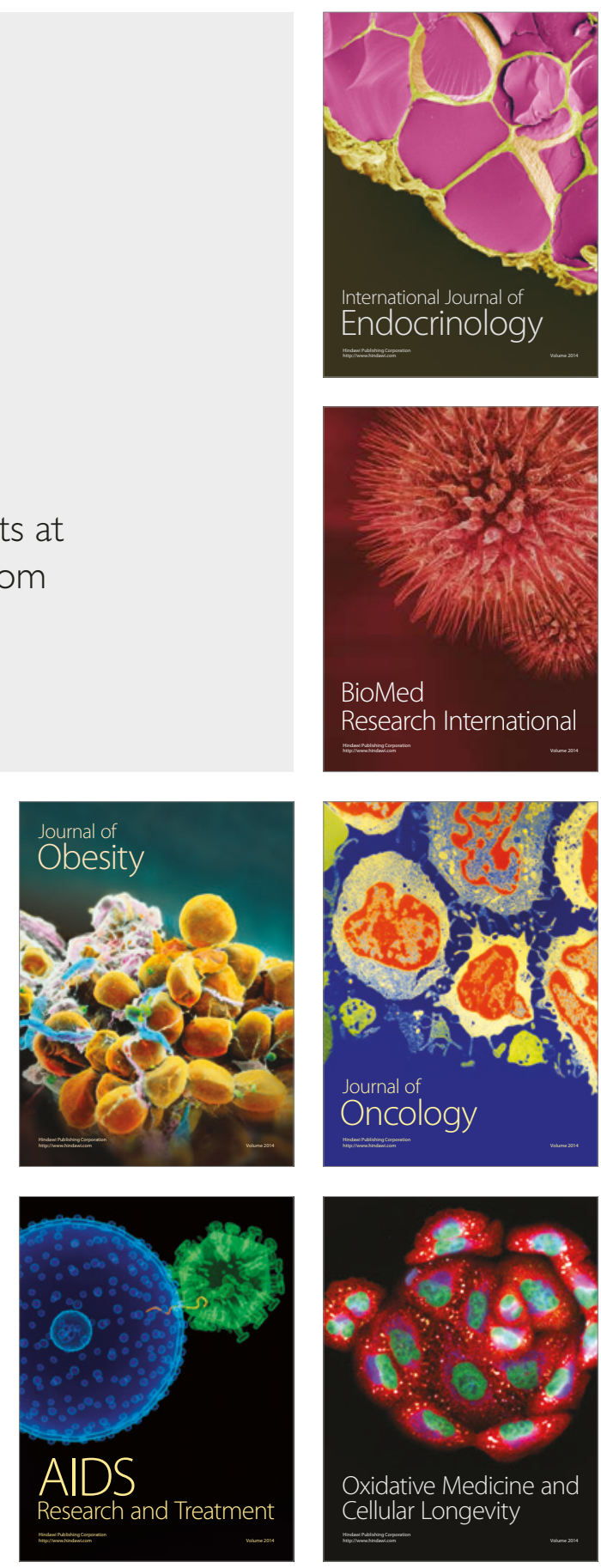